\title{
A MODEL OF THE JOVIAN INTERNAL FIELD DERIVED FROM IN-SITU AND AURORAL CONSTRAINTS
}

\author{
S. L. G. Hess*, B. Bonfond`, F. Bagenal ${ }^{\ddagger}$ and L. Lamy ${ }^{\S}$
}

\begin{abstract}
The internal magnetic field of Jupiter is known to be highly multi-polar, not only from the direct measurements performed by the Voyager and Pioneer probes but also from the unusually complex shape of the northern auroral oval. The limited amount of data obtained from the Voyager and Pioneer flybys do not permit accurate determination of the topology of the magnetic field, as they barely constrain, even the octupole contribution to the field. This does not allow one to reproduce the position of the auroras nor satisfactorily explain the shape and frequency range of the Jovian radio arcs. Successive attempts have been made to constrain the higherorder field using the position of the Io auroral footprint where the auroras are due to currents generated close to Io and carried along the magnetic field lines. Thus, the auroral spots should map to Io's orbit. VIPAL, the latest model of this kind is a 5th order model. However, the VIPAL model was limited by three factors: the main constraints come from a unique L-Shell, the difficulty of mixing jovigraphic and magnetic data, and the non-linearity of the problem. These issues lead to numerically-demanding computations, with the scale of computation increasing as the square of the model order. We have developed a new method for computing the magnetic field using in-situ and auroral constraints (ISaAC) which we have applied to the computation of the Jovian magnetic field, based on Voyager, Pioneer, Galileo magnetic measurements and constrained by Io's, Europa's and Ganymede's auroral footprint locations.
\end{abstract}

\section{Introduction}

The dynamics of Jupiter's metallic interior generates the most intense planetary magnetic field of our solar system, with a magnetic moment of about $4 G \cdot R_{J}^{3}\left(1 R_{J}=71492 \mathrm{~km}\right)$,

\footnotetext{
*ONERA - The French Aerospace Lab, 2 Avenue Edouard Belin, 31400 Toulouse, France

${ }^{\dagger}$ Space sciences, Technologies and Astrophysics Research (STAR) Institute, LPAP, Université de Liège, Liège, Belgique

${ }^{\ddagger}$ LASP, University of Colorado, Boulder, USA

${ }^{\S}$ LESIA
} 
i.e. almost 18000 times Earth's field.The magnetic field of Jupiter, as the magnetic fields of all planets with the exception of the Earth, was first modeled from the magnetic field measurements performed by spacecraft flying by the planet. In the case of Jupiter, the Pioneer 10 and 11 [Acuna and Ness, 1975b], and Voyager 1 [Ness et al., 1979a] measurements provided the most important constraints on the magnetic field determination [Connerney, 1993]. Additional data were obtained by the Voyager 2 [Ness et al., 1979b], Ulysses [Balogh et al., 1992] and Galileo flyby, but imposed fewer constraints as a result of the larger approach distances of these spacecraft to Jupiter.

The strong magnetic moment creates a large magnetosphere around the planet, within which magnetic fields, internal plasma and satellites interact, generating currents which in turn generate perturbations of the magnetic field. The magnetospheric magnetic field $(B)$ can be split into a planetary internal magnetic field, which derives from a potential field $(V)$, and a magnetic perturbation $(b)$ due to magnetospheric currents:

$$
B=-\nabla V+b
$$

The expression for the magnetospheric perturbation $(b)$ is derived from magnetospheric current observations and modeling but remains poorly known [Connerney et al., 1981; Khurana, 1997; Khurana and Schwarzl, 2005]. Moreover these currents are likely to change with conditions in the magnetosphere [Grodent et al., 2008b]. The two components of the magnetic field should therefore be computed together in order to achieve a self-consistent model. However, most of the internal magnetic field models computed so far rely on the Connerney et al. [1981] current sheet model which itself relies of the Pioneer's era O4 internal magnetic field model. As long as the later models have low order coefficients that are close to those of the $\mathrm{O} 4$ model then this is not a problem, but this condition is not always satisfied. The most accurate magnetic field model constructed only using the inversion of magnetic field measurements is the O6 magnetic model Connerney et al.

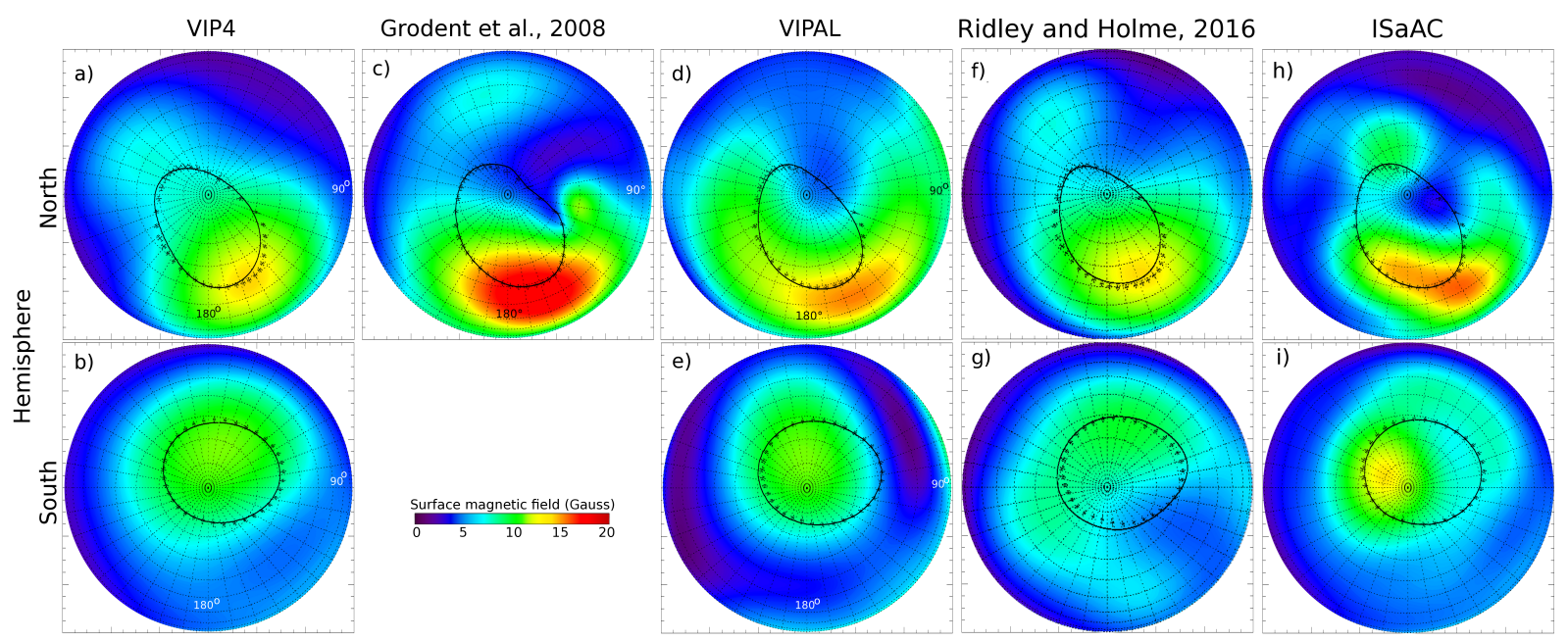

Figure 1: Surface magnetic field for different magnetic field models. Ridley and Holme [2016] is the non-drifting (JCF) damped $\left(\lambda=2.10^{-10}\right)$ model which coefficients are provided in the paper. The observed Io footprints and the modeled Io footpaths are overplotted on the figures. 
[1996]. Another model was recently computed [Ridley and Holme, 2016] allowing for a secular variation of the moments, but the method used does not provide a unique solution.

Field aligned currents that are induced by the magnetosphere interactions follow the magnetic field lines and close in the Jovian ionosphere, giving rise to intense auroral emissions. Hence, the locations of the auroras on top of the Jovian ionosphere must map to the regions of the magnetosphere where the interactions take place. In the case of the magnetosphere interaction with a satellite, this correspondence can be verified with a high accuracy, since the interaction is highly localized and the position of the satellite precisely known.

The most intense satellite-driven auroras are those due to Io's interaction with the Jovian magnetosphere. The UV auroral signature of Io consist of a main spot, secondary spots and an extended tail at the footprints of the magnetic field line carrying the current generated by Io's interaction with the magnetosphere [Bonfond et al., 2012]. This substructure of the footprint is interpreted as being due to the Alfvénic current system at Io [Neubauer, 1980; Saur, 2004]: As the current-carrying Alfvén waves generated at Io travel toward Jupiter, they are partially reflected and dispersed, which produces the observed spots [Hess et al., 2010b; Jacobsen et al., 2007]. The main spot is related to the most direct trajectory of the current-carrying Alfvén waves, hence the associated magnetic field lines should map close to Io's position.

Many studies of the magnetic mapping of Io to the planet and its comparison with the positions of Io's footprints observed in UV and Infrared have been performed [Connerney et al., 1998; Grodent et al., 2008a; Bonfond et al., 2009], which have led to the determination of new internal magnetic field models : The VIP4 model Connerney et al. [1998] derived from early IR observation of the Io footpath and Pioneer and Voyager measurements and the VIT4 internal magnetic model [Connerney, 2007] developed using mostly the position of the Io footpaths and the azimuthal component of the magnetic field measured by Voyager. In order to obtain a better fit of the satellite footpaths, in particular the kink in the Io northern footpath near a longitude of $110^{\circ}$, Grodent et al. [2008a] proposed to add a magnetic anomaly modeled by a supplementary dipole. However, none of these models allowed a correct longitudinal mapping of the Io footprints: Studies by Bonfond et al. [2009] in the UV and Hess et al. [2010a] in radio showed a large (up to $10^{\circ}$ ) longitude difference between the predicted and observed positions of the Io footprints.

More recently, a statistical investigation of the Ganymede and Europa UV footprints has also been performed [Clarke et al., 2002; Grodent et al., 2006, 2009; Bonfond et al., 2013]. Grodent et al. [2008a] gives a table of the Europa and Ganymede footprint locations as a function of the longitude of the satellites, which were not mapped by previous models. Hess et al. [2011] developed a new model mainly based on the Io footprint location mapping that allowed to retrieve the correct location (in longitude and latitude) of the northern and southern Io footprints and that improved the mapping of Europa and Ganymede footpath. However, this model was not able to correctly reproduce the magnetic field measurements for L-Shells smaller than $4 R_{J}$. This problem arose from 

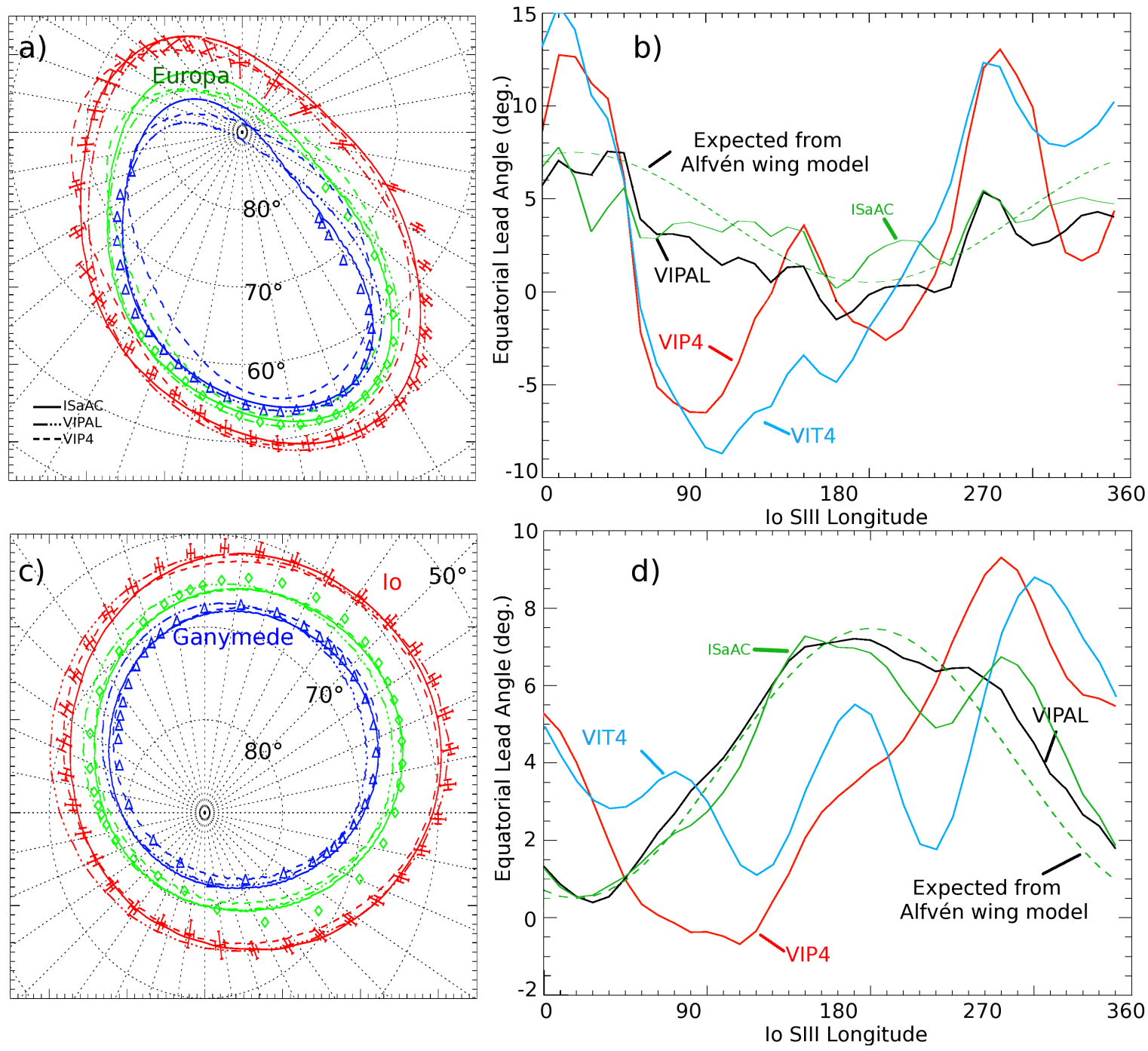

Figure 2: Mapping of the Io, Europa and Ganymede footpaths in the northern (a) and southern (c) hemispheres with ISaAC, VIPAL and VIP4. Lead angle of the Io footprint versus Io's longitude with in the northern (b) and southern (d) hemispheres.

the method used to derive the magnetic field model that was mostly based on the fit of the shape of the $L \simeq 6 R_{J}$ shell, with a minimal contribution from the magnetic field measurements closer to Jupiter.

\section{Data}

\subsection{Magnetic measurements}

The most constraining measurements of the magnetic field close to Jupiter have been performed by the Voyager 1 magnetometer (MAG) [Behannon et al., 1977] and the Pioneer 10 \& 11 Helium Vector Magnetometer (HVM) [Acuna and Ness, 1975a]. These three flybys are the closest ever made to Jupiter before JUNO. Measurements close to the 
planet avoid, in part, the effect of the strong current-sheet magnetic field, and constrain higher orders of the spherical harmonics decomposition of the internal magnetic field. However, these three flybys cover a very restricted region of the magnetosphere and give only sparse measurements of the magnetic field versus longitude, latitude and distance [Connerney, 1981]. In the present paper, we use the Ulysses [Balogh et al., 1992] and Galileo measurements as a complement to those of Voyager and Pioneer to determine our model. These measurements bring better constraints on the free parameters. Before using the Voyager and Pioneer measurements to constraint our model, it is necessary to first subtract the magnetic field generated by the current sheet. The current sheet magnetic field is computed using the Connerney [1981] model.

In order to estimate the accuracy of the fit of the magnetic field, it is necessary to pay attention to the statistical weight of each measurement: two measurements performed in very different regions are more constraining than two measurements performed at the same location. We organized the measurements by hemisphere and bins of $10^{\circ}$ in longitude and $0.35 R_{J}$ in L-shell. Each measurement weight is the inverse of the number of measurements in its bin. In the present paper, we used only the measurements performed closer than 10 $R_{J}$ to Jupiter, i.e. in a region where the current sheet is well described.

\subsection{Auroral footprints}

The Io auroral footprint data that are used in the present paper are the same than those used for deriving the VIPAL model [Hess et al., 2011]. Ganymede and Europa footprint data that were used in the present paper are also provided by Hess et al. [2011]. Only Io's lead angle was fitted, using the theoretical lead angles provided in Hess et al. [2011]. The average lead angle is more difficult to constrain from the observation than the amplitude of its variation and is a free parameter. The fact that the average lead angle must be the same for both hemisphere is enforced:

$$
\alpha_{N} \simeq A-3.5^{\circ} \cos \left(\lambda_{I o}-20^{\circ}\right)
$$

The model computation determined $A \simeq 4^{\circ}$ which was the value expected in [Hess et al., 2011]. This value is the same for both hemispheres and prevents the nonphysical negative lead angles found with the VIPAL model.

Europa and Ganymede lead angles were not used for two reasons. Their theoretical lead angles are less constrained than Io's. Moreover, the variation of the position of the footprints in [Hess et al., 2011] is not consistent with the motion of a single spot, but more probably mixes the positions of different spots. Indeed, multiple footprints of Ganymede were found in latter observations [Bonfond et al., 2013]. 


\section{Magnetic field modeling}

The internal potential field $V$ is usually expressed in term of spherical harmonics Connerney [1981]:

$$
V=\sum_{n} \sum_{m} \frac{P_{n}^{m}(\cos \theta)\left[g_{n}^{m} \cos (m \phi)+h_{n}^{m} \sin (m \phi)\right]}{r^{n+1}}
$$

where $P_{n}^{m}$ is the Schmidt-normalized Legendre function of degree $n$ and order $m$, and the $g_{n}^{m}$ and $h_{n}^{m}$ terms are the Schmidt coefficients describing the planet's internal magnetic field, and $r$ is the distance relative to the planet's center, normalized to the Jovian equatorial radius. In the ideal case, where accurate data are available with a regular longitudinal and latitudinal spacing at a constant and low altitude, this is similar to a simple 2D Fourier transform and simple to compute. This technique will be applied to the JUNO data once the probe has finished mapping the Jovian near-surface magnetic field. Using sparser and less regular measurements such as those obtained from Pioneer and Voyager flybys lead to a computation limited to low orders and/or to non-unique or poorly constrained determination of some high order coefficients.

The model corresponding to the solution that is both unique and of the highest possible order is the $\mathrm{O} 6$ model. In this case the coefficient have a physical meaning in the sens that they can be exploited to study geophysical processes in the Jovian interior [Duarte et al., 2016]. But models built from the fit of the Io footpath do not share this property: in this case coefficients shall be seen as pure mathematical coefficients with no physical meaning. This is particularly true for the VIPAL model which was primarily built on the fit to the boundaries of the $L=6 R_{J}$ shell. As highlighted by the authors, this fit constrains some linear combinations of the coefficients, but not some others. As an example, for VIP4 and VIT4 $g_{2}^{0}+g_{4}^{0} \simeq-0.2 G$ and $g_{2}^{0}-g_{4}^{0} \simeq-0.15 G$ while for VIPAL $g_{2}^{0}+g_{4}^{0} \simeq-0.1 G$ and $g_{2}^{0}-g_{4}^{0} \simeq 1.4 G$. If the sum of the two coefficients is relatively well constrained, their difference is not.

The limitations of the VIPAL model came from the method used to compute it: starting from the VIP4 model, the authors found the position of the Io footprints along the footpath as a function of Io's longitude by successive modifications of only the $g_{m}^{m}, g_{m+1}^{m}, h_{m}^{m}$ and $h_{m+1}^{m}$ coefficients (as in this case the system has a unique solution). Then using successive modifications of all of the 35 coefficients, they performed a minimization of a linear combination of the errors on the fit of the Io footprint positions in longitude and latitude and of the magnetic field measurements (all normalized by their respective error bars). Successive modifications of the coefficients was used instead of computing a covariance matrix to save computational time. Building the full matrix requires computing the variation of the quantity to minimize as a function of of all coefficients. To fit the footprints, this is done by rebuilding all magnetic field lines for each coefficient variation which is a long process for higher order models (the number of computations increasing as the square of the order). 


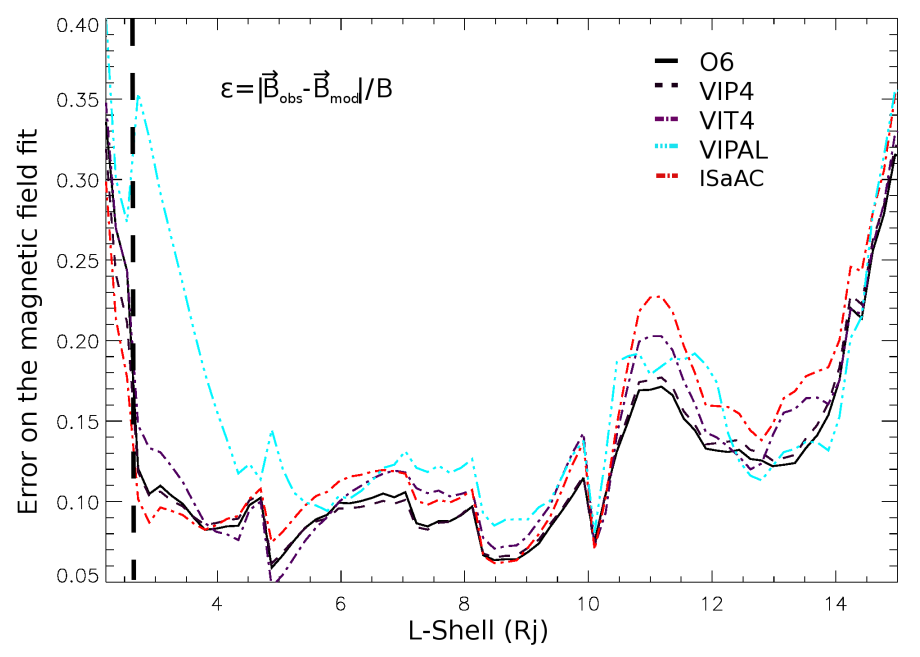

Figure 3: RMS error on the fit of the magnetic vector as a function of the L-Shell. Below 2.5 $R_{J}$, the error increases due an increasing error on the attitude of the spacecraft during the data acquisition.

In the present paper, we simplified the computation by (1) computing the 3D magnetic shells corresponding to Io, Europa and Ganymede orbits using a precise algorithm (with small spatial steps); (2) under-sampling them using 2880 points (36 field lines separated by $10^{\circ}$ at equator, 80 points per field line); (3) estimating on each point the magnetic field vector derivative versus the coefficient variations; (4) estimating the field line deformations from these derivatives (assuming that the field line deformation is small enough so that the variation of the magnetic field vectors computed along the initial field line applies along the deformed one); (5) computing the derivative of the footprint position from the field line deformations; (6) building the covariance matrix from estimates of the footprint position and magnetic field vector variations; (7) perform a singular value decomposition of the matrix, filter the under-constrained coefficients and invert the matrix as in Connerney [1981]; (8) updating the coefficients.

This method is iterative: at each iteration we perform only one computation of the field lines at full precision (with a spatial step down to $70 \mathrm{~km}$ at the surface), $(m+1)^{2}-1$ computations of the shell deformations due to the coefficient variations (with $m$ the order of the model), and one matrix inversion. It is noteworthy that the precise field line computation take $80 \%$ of the computational time. In the original method [Connerney, 1981], $2\left((m+1)^{2}-1\right)$ computations of the field lines were performed to get the position derivatives, but the precision to reach was weaker (due to the largest error bars on the measurements), the computations were limited to the Io field lines and the order limited to the $4^{\text {th }}$ (i.e. 24 coefficients). In the $5^{\text {th }}$ order VIPAL case, there were also $2\left((m+1)^{2}-1\right)$ computations of the field lines with a high precision, but the algorithm converged much faster than those using matrix inversion, although not toward the optimal solution. 


\section{Results}

With this method, we are able to compute in reasonable times magnetic field models of the $10^{\text {th }}$ order (120 coefficients) using Io, Europa and Ganymede footprint positions. The method was run until it converged, with no reworking of the final coefficients to remove those that cannot be accurately constrained. Thus high order coefficients should not be considered as physically meaningful. The method was first run up to the eighth order, and in a second time to the tenth order. Typically coefficients up to the sixth order remain unchanged when higher orders are added, which suggests that they are correctly constrained. Even though, these coefficients correspond for a large part to the fit of the footpath of the L-Shells of Io, Europa and Ganymede, i.e. to a rather small latitudinal range. They should not be used, therefore, for studying the internal structure of Jupiter. A study of the significance of the coefficients was not performed because JUNO's data will provide a definitive Jovian magnetic field model that will be much better constrained and accurate than the present one. The main purpose of present model is to be an engineering model to perform studies requiring an accurate modeling of the magnetic field line topology and to process JUNO data before the final JUNO model is published. The secondary interest of the present model would be to benchmark the method. However the inaccuracy on the Jovian rotation period coupled to the use of data acquired more than 30 years apart prevents us to expect an accurate comparison with the JUNO model at high orders. The 120 coefficients of the model are presented in Table 1.

Figure 1 shows the radial surface magnetic fields for different models. One can see that models with high order tend to present a more complex surface field. More interestingly, although these models may be obtained from very different method, the overall morphology of the radial field on the surface tends to be the same, confirming the presence of a magnetic anomaly in the northern hemisphere. Moreover, Ridley and Holme [2016] show in their paper the surface field corresponding to a less damped constant field that resembles even more to that obtained with ISaAC (see discussions in Ridley and Holme [2016]).

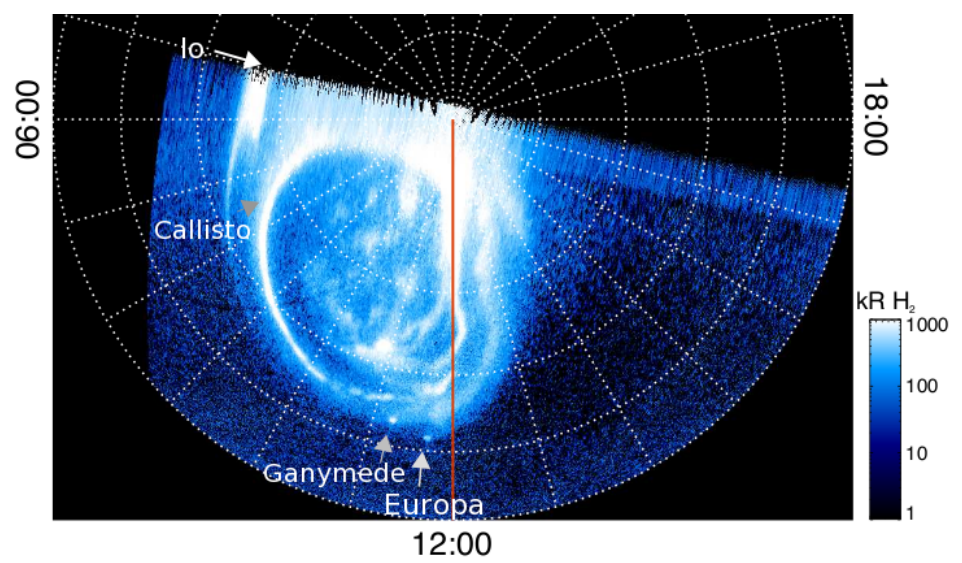

Figure 4: Observation in the UV of the Jovian northern auroras made by Hubble and obtain through the APIS service. Arrows show the predicted locations of the four Galilean satellite footprints. 
Figure 2 shows the fits of Io, Europa and Ganymede footpaths. The match to the Ganymede northern footpath is significantly improved with the new model, showing the typical bean shape of Jupiter's northern auroral oval. ISaAC is used systematically in the APIS web-service which archives and provides UV observations of Jupiter since 2015 [Lamy et al., 2015] to provide markers of the Galilean footprints on top of UV polar projections (Fig. 4). The match to the longitude of the Io footprints id slightly worse than for the VIPAL model, but the present method corrects the apparent $2^{\circ}$ shift in longitude between the northern and the southern footprints which could not be addressed by the computational method used to determine the VIPAL model.

Figure 3 shows the error on the fit of the magnetic field measurements. ISaAC performance is comparable with that of VIP4, and much better than VIPAL for close L-Shells. This is due to a better handling of the magnetic field data in the present method compared to the VIPAL method.

Table 1: Model Schmidt coefficients

\begin{tabular}{|c|c|c|c|c|c|c|c|c|c|c|c|}
\hline$h^{m}$ & 1 & 2 & 3 & 4 & 5 & 6 & 7 & 8 & 9 & 10 & $g_{n}$ \\
\hline & & & & & & & & & & & $\overline{g^{m}}$ \\
\hline & 4.0665 & -0.1286 & -0.0479 & -0.2230 & -0.0165 & -0.0637 & 0.0064 & -0.0511 & 0.0010 & 0.0028 & 0 \\
\hline & -0.7142 & -0.6981 & -0.4642 & 0.1893 & 0.0755 & 0.0324 & 0.0892 & 0.0720 & 0.0033 & 0.0049 & 1 \\
\hline & & 0.3852 & 0.2867 & 0.0276 & 0.0623 & 0.0802 & 0.0366 & 0.0453 & -0.0096 & -0.0063 & 2 \\
\hline$h_{n}$ & & & -0.0934 & -0.1317 & -0.0150 & 0.0027 & -0.0698 & -0.0371 & 0.0037 & 0.0091 & 3 \\
\hline 1 & 0.2353 & & & 0.0111 & -0.0400 & -0.0407 & -0.0114 & -0.0020 & 0.0339 & 0.0285 & 4 \\
\hline 2 & -0.3170 & 0.0795 & & & 0.0142 & 0.0279 & 0.0239 & 0.0312 & -0.0074 & -0.0038 & 5 \\
\hline 3 & -0.07503 & 0.4031 & -0.3686 & & & -0.0068 & -0.0123 & -0.0210 & -0.0068 & -0.0062 & 6 \\
\hline 4 & 0.2078 & 0.3293 & -0.1695 & 0.0596 & & & 0.0031 & 0.0071 & 0.0040 & 0.0044 & 7 \\
\hline 5 & 0.0003 & -0.0334 & 0.0254 & 0.0349 & -0.0084 & & & -0.00133 & -0.00092 & -0.00169 & 8 \\
\hline 6 & 0.1051 & -0.0960 & 0.0503 & 0.0127 & -0.0106 & 0.0018 & & & 0.00009 & 0.00031 & 9 \\
\hline 7 & -0.0027 & -0.0297 & 0.0739 & 0.0198 & -0.0212 & 0.0035 & 0.00019 & & & -0.000008 & 10 \\
\hline 8 & 0.0781 & -0.0738 & 0.0852 & -0.0087 & -0.0168 & 0.0072 & -0.00008 & -0.00046 & & & \\
\hline 9 & -0.0029 & 0.0189 & 0.0185 & 0.0069 & 0.0032 & -0.0043 & 0.0027 & -0.0012 & 0.00027 & & \\
\hline 10 & 0.0023 & 0.0172 & 0.0125 & -0.0011 & -0.0011 & -0.00015 & 0.0036 & -0.0025 & 0.0008 & -0.00014 & \\
\hline
\end{tabular}

\section{References}

Acuna, M. A., and N. F. Ness, The Pioneer XI high field fluxgate magnetometer, Space Science Instrumentation, 1, 177-188, 1975a.

Acuna, M. H., and N. F. Ness, Jupiter's main magnetic field measured by Pioneer 11, Nature, 253, 327-+, 1975b.

Balogh, A., M. K. Dougherty, R. J. Forsyth, D. J. Southwood, E. J. Smith, B. T. Tsurutani, N. Murphy, and M. E. Burton, Magnetic field observations during the ULYSSES flyby of Jupiter, Science, 257, 1515-1518, doi:10.1126/science.257.5076.1515, 1992.

Behannon, K. W., M. H. Acuna, L. F. Burlaga, R. P. Lepping, N. F. Ness, and F. M. Neubauer, Magnetic field experiment for Voyagers 1 and 2, Space Science Review, 21, 235-257, 1977. 
Bonfond, B., D. Grodent, J.-C. Gérard, A. Radioti, V. Dols, P. A. Delamere, and J. T. Clarke, The Io UV footprint: Location, inter-spot distances and tail vertical extent, Journal of Geophysical Research (Space Physics), 114(A13), 7224-+, doi: 10.1029/2009JA014312, 2009.

Bonfond, B., D. Grodent, J.-C. Gérard, T. Stallard, J. T. Clarke, M. Yoneda, A. Radioti, and J. Gustin, Auroral evidence of Io's control over the magnetosphere of Jupiter, Geophys. Rev. Letter, 39, L01105, doi:10.1029/2011GL050253, 2012.

Bonfond, B., S. Hess, F. Bagenal, J.-C. Gérard, D. Grodent, A. Radioti, J. Gustin, and J. T. Clarke, The multiple spots of the Ganymede auroral footprint, Geophys. Rev. Letter, 40, 4977-4981, doi:10.1002/grl.50989, 2013.

Clarke, J. T., et al., Ultraviolet emissions from the magnetic footprints of Io, Ganymede and Europa on Jupiter, Nature, 415, 997-1000, 2002.

Connerney, J. E. P., The magnetic field of Jupiter - A generalized inverse approach, Journal of Geophysical Research, 86, 7679-7693, doi:10.1029/JA086iA09p07679, 1981.

Connerney, J. E. P., Magnetic fields of the outer planets, Journal of Geophysical Research, 98, 18,659-+, doi:10.1029/93JE00980, 1993.

Connerney, J. E. P., Planetary Magnetism, vol. 10: Planets and Satellites, pp. 243-280, Treatise in Geophysics, eds. G. Schubert, T. Spohn, Elsevier, Oxford, UK, 2007.

Connerney, J. E. P., M. H. Acuna, and N. F. Ness, Modeling the Jovian current sheet and inner magnetosphere, Journal of Geophysical Research, 86, 8370-8384, doi: 10.1029/JA086iA10p08370, 1981.

Connerney, J. E. P., M. H. Acuña, and N. F. Ness, Octupole model of Jupiter's magnetic field from Ulysses observations, Journal of Geophysical Research, 101, 27,453-27,458, doi:10.1029/96JA02869, 1996.

Connerney, J. E. P., M. H. Acuña, N. F. Ness, and T. Satoh, New models of Jupiter's magnetic field constrained by the Io flux tube footprint, J. Geophys. Res., 103(12), 11,929-11,940, doi:10.1029/97JA03726, 1998.

Duarte, L. D. V., J. Wicht, and T. Gastine, Physical conditions for Jupiter-like dynamo models, ArXiv e-prints, 2016.

Grodent, D., J. Gérard, J. Gustin, B. H. Mauk, J. E. P. Connerney, and J. T. Clarke, Europa's FUV auroral tail on Jupiter, Geophysical Research Letter, 33, 6201-+, doi: 10.1029/2005GL025487, 2006.

Grodent, D., B. Bonfond, J. Gérard, A. Radioti, J. Gustin, J. T. Clarke, J. Nichols, and J. E. P. Connerney, Auroral evidence of a localized magnetic anomaly in Jupiter's northern hemisphere, Journal of Geophysical Research (Space Physics), 113(A12), 9201-+, doi:10.1029/2008JA013185, 2008a. 
Grodent, D., J. Gérard, A. Radioti, B. Bonfond, and A. Saglam, Jupiter's changing auroral location, Journal of Geophysical Research (Space Physics), 113(A12), 1206-+, doi:10.1029/2007JA012601, 2008b.

Grodent, D., B. Bonfond, A. Radioti, J.-C. Gérard, X. Jia, J. D. Nichols, and J. T. Clarke, Auroral footprint of Ganymede, Journal of Geophysical Research (Space Physics), 114(A13), 7212-+, doi:10.1029/2009JA014289, 2009.

Hess, S., A. Petin, P. Zarka, B. Bonfond, and B. Cecconi, Lead angles and emitting electron energies of Io-controlled decameter radio arcs, Planetary and Space Sciences, 58, 1188-1198, doi:doi:10.1016/j.pss.2010.04.011, 2010a.

Hess, S. L. G., P. Delamere, V. Dols, B. Bonfond, and D. Swift, Power transmission and particle acceleration along the Io flux tube, Journal of Geophysical Research (Space Physics), 115(A14), 6205-+, doi:10.1029/2009JA014928, $2010 \mathrm{~b}$.

Hess, S. L. G., B. Bonfond, P. Zarka, and D. Grodent, Model of the Jovian magnetic field topology constrained by the Io auroral emissions, Journal of Geophysical Research (Space Physics), 116, A05,217, 2011.

Jacobsen, S., F. M. Neubauer, J. Saur, and N. Schilling, Io's nonlinear MHD-wave field in the heterogeneous Jovian magnetosphere, Geophys. Res. Letters, 34, 10,202-+, doi: 10.1029/2006GL029187, 2007.

Khurana, K. K., Euler potential models of Jupiter's magnetospheric field, Journal of Geophysical Research (Space Physics), 102, 11,295-11,306, doi:10.1029/97JA00563, 1997.

Khurana, K. K., and H. K. Schwarzl, Global structure of Jupiter's magnetospheric current sheet, Journal of Geophysical Research (Space Physics), 110(A9), 7227-+, doi: 10.1029/2004JA010757, 2005.

Lamy, L., R. Prangé, F. Henry, and P. Le Sidaner, The Auroral Planetary Imaging and Spectroscopy (APIS) service, Astronomy and Computing, 11, 138-145, doi: 10.1016/j.ascom.2015.01.005, 2015.

Ness, N. F., M. H. Acuna, R. P. Lepping, L. F. Burlaga, K. W. Behannon, and F. M. Neubauer, Magnetic field studies at Jupiter by Voyager 1 - Preliminary results, Science, 204, 982-987, 1979a.

Ness, N. F., M. H. Acuna, R. P. Lepping, L. F. Burlaga, K. W. Behannon, and F. M. Neubauer, Magnetic field studies at Jupiter by Voyager 2 - Preliminary results, Science, 206, 966-972, 1979b.

Neubauer, F. M., Nonlinear standing Alfven wave current system at Io - Theory, J. Geophys. Res., 85(.14), 1171-1178, 1980.

Ridley, V. A., and R. Holme, Modeling the Jovian magnetic field and its secular variation using all available magnetic field observations, Journal of Geophysical Research (Planets), 121, 309-337, 2016. 
Saur, J., A model of Io's local electric field for a combined Alfvénic and unipolar inductor far-field coupling, Journal of Geophysical Research (Space Physics), 109(A18), 1210-+, doi:10.1029/2002JA009354, 2004. 